\title{
Im Zwiespalt
}

\author{
Die Balance ist weg. Aus Datenschutzgründen stehen Pandemieforscher \\ immer wieder vor gesperrten Datensätzen. Dabei ist die Wissenschaft ausschließlich \\ an anonymisierten Informationen interessiert. \\ Von Josef Ruhaltinger
}

Niki Popper hat in der Wahrnehmung der Österreicher eine Steilkurve hingelegt. Seine lockeren Auftritte bei zahllosen Regierungs-Pressekonferenzen, Talk-Runden der Stöckl-Sisters bis hin zu Doppelseiten in der Krone Bunt bescherten dem Zahlenmenschen eine Öffentlichkeit, deren Intensität sich gerade noch hinter Hermann Maier verorten lässt. Für einen Menschen, dessen Hauptgesprächsthema Zahlenmodelle sind, ist dies eine unerwartete Publicity.

Niki Popper spielt nicht mit der Rolle des sympathischen Nerds. Er ist einer. Man möchte mit ihm auf ein Bier gehen. Und dies auch dann, wenn man nicht alles versteht, wovon er redet. Er ist ein Meister der Selbstironie. In der Talk Show von Barbara Stöckl bezeichnet er seinen und Michael Niavaranis Auftritt als einen von „zwei bladen Glatzerten“. Und weil der Kabarettist und der Statistikmensch sich gefunden hatten, vereinbarten sie für einen späten Septemberabend - 26.9., um genau zu sein - einen gemeinsamen Bühnenabend in Niavaranis "Theater im Park“. Titel der Doppel-Conference: „Zahlen, bitte!“

Seine öffentliche Präsenz bringe Nutzen, behauptet Popper. „Wenn ich mich ins Mittagsjournal stelle und behaupte, wir benötigen spezielle Daten, dann erziele ich einen höheren Impact als noch vor zwei Jahren." Und der Datenhunger sei groß: „Wir entwickeln seit zehn Jahren ein Modell, das in der Lage ist, für Simulationen und Prognosen sehr viele unterschiedliche Daten zu integrieren." Und dies erlaube vielerlei Einsatzszenarien. Voraussetzung: „Die Daten müssen in professionell anonymisierter Form zur Verfügung stehen."

Niki Popper definiert damit den Flaschenhals der heimischen Datenanalyse: Nicht die Datenmenge ist das Problem. Die Informationen liegen auf vielen, vielen Speichern über Österreich verstreut. Aber zu viele Institutionen und Behörden sind in Österreich nicht in der Lage, Daten für Analysten in rechtskonformer und nutzbarer Weise zu liefern - mit großen Auswirkungen auf die Qualität von Entscheidungsgrundlagen und deren Auswirkungen.

\section{Uneinheitliche Datenquellen}

Die Wogen gehen hoch, wenn Datennutzer mit den Grenzen des Datenschutzes konfrontiert werden. Der emeritierte Statistikprofessor der Universität Wien, Erich Neuwirth, klagt in vielteiligen Beiträgen auf Twitter, dass Spitalsdaten nicht gegliedert nach Impfstatus vorlägen. Dadurch gäbe es keine Rückschlüsse über „die Impf-Wirkung bei schwereren Erkrankungen. Und wir wissen nicht, wie lange nach der Impfung es wie oft zu schweren COVID-Verläufen kommt." Als Datenexperte fordert Neuwirth, man müsse nur die Daten aus dem e-Impfpass mit den Spitalsdaten verknüpfen. Aber dies sei aus Gesetzesgründen nicht erlaubt. Er stelle sich die Frage, „ob es eine Entscheidung der Bundesländer sein soll, welche Daten geliefert werden und welche nicht“. Neuwirth ortet einen „Kompetenzmangel bei den Leuten, die diese Daten aufbereiten“.

Die Nutzung von Gesundheitsdaten ist - auch im öffentlichen Auftrag - ein sensibler Bereich. In Österreich äußert sich dies im extrem langsamen Fortschritt digitaler eHealth-Projekte. Das eRezept, mit dem der Arzt auf elektronischem Weg Rezepte verschreiben kann, ist nach fünf Jahren Entwicklungszeit immer noch nicht im Roll-out. Und die persönliche elektronische Gesundheitsakte, die Mutter aller eHealth-Services, ist unverändert Stückwerk, deren Einführung im niedergelassenen Bereich nur sehr zögerlich angenommen wird (siehe Interview mit ELGA-Geschäftsführer Franz Leisch auf Seite 8).

Die Pandemie sorgte unversehens für Druck. Das Virus führte Österreichs Entscheidern vor Augen, dass Informationen über Ausbreitung, Hospitalisierungen, Zuwachsraten, Ursprünge oder Verteilungen von Krankheitsfällen nur sehr unzulänglich vorhanden waren. Niki Popper: „Wir haben bei Covid-19 gesehen: Ohne Auswertungen stehen wir nackert da." Faktenbasierte Entscheidungen blieben mangels Grundlagen während der ersten Welle die Ausnahme. Die Auswirkungen sorgten für enorme Verunsicherung in der Bevölkerung. Eine der zentralen Ursachen des heimischen Schlingerkurses in der Pandemiebekämpfung liegt im föderal organisierten Gesundheitssystem Österreichs. Die Länderkompetenz in Sachen Gesundheit steht und stand in Widerspruch zum Vorteil von zentralisierten Datensammelstellen. Das Konzept von ELGA ist ein Spiegelbild des Systems: Die Speicherung der Daten von Entlassungsbriefen, Laborbefunden und Bildbefunden erfolgt dezentral in den Bundesländern - entweder in den 13 ländereigenen Datenspeichern oder direkt in jenen Organisationen, in denen sie entstehen. Das sind in erste Linie Spitäler, und - mit Abstrichen - Labore und Röntgenordinationen. Der überwiegende Rest des niedergelassenen Bereichs zaudert, seine Befunde in das ELGA-System einzuspeisen. Als Hauptgründe werden Datenschutzargumente angeführt.

\section{Angriffsziel Krankenhaus}

Christoph Drescher versteht die Skepsis breiter Bevölkerungsschichten. „Gesundheitsdaten haben für Kriminelle einen hohen Wert. Der Schaden bei lahmgelegten Gesundheitseinrichtungen oder bloßgestellten Patientendaten ist immens. Und die Gefahr eines Hackerangriffs ist bei hohen Lösegeldsummen sehr real." Christoph Drescher ist Managing Partner bei dem Wiener Startup MyPrivacy, das eine Kryptografie-Lösung entwickelt hat, um vertrauliche Daten sicher mit externen Empfängern zu teilen. Der Schutz von Daten ist sein tägliches Brot.

Die internationale IT-Mafia hat den Gesundheitsbereich längst als Angriffsziel ausgemacht. Erst im Oktober vergangenen 
Jahres ist mit Universal Health Services (UHS) einer der größten US-Gesundheitsdienstleister (400 Spitäler in US und GB) von einer Ransomware-Attacke heimgesucht worden. Alle Daten auf den Computern wurden verschlüsselt und Zugang wird erst wieder gewährt, wenn Lösegeld bezahlt wurde - oder die Einrichtung ein Backup der Daten einspielt.

Im Mai des Jahres ist das Ireland's Health Service HSE Ziel einer ähnlichen Attacke geworden. Der irische Gesundheitsdienst musste sein gesamtes Computer-System abschalten. Der Angriff ziele auf IT-Anlagen, auf denen Patientendaten gespeichert seien, sagte der Chef einer betroffenen Entbindungsklinik.

IT-Angriffe gehören heute zum Alltag - egal ob im privaten Homeoffice, hinter Firewalls von Unternehmen, Bankensystemen oder in den Serverkellern der (Gesundheits)-Behörden. Für Christoph Drescher wird die Abwägung zwischen dem Schutz der Privatsphäre und der Nutzung von persönlichen Gesundheitsdaten von der Absicht bestimmt, was mit den Informationen gemacht werden soll: „Es kommt immer darauf an, was auf dem Spiel steht.“ Pandemieforschung sei ein „hehres Anliegen, bei dem die Gewichtung des öffentlichen Interesses hoch einzuschätzen sei“. Aber die „lückenlose und unveränderbar dokumen- tierte, gesetzeskonforme Verarbeitung“ bleibe unverzichtbare Voraussetzung.

\section{Neue Prioritäten}

Die Pandemie hat die vorsichtige Haltung der Politik gegenüber dem Datenschutz verändert. Mit der e-Medikation und mit dem Impfregister wurden zuletzt zwei große zentrale Datenspeicher für Gesundheitsdaten eingerichtet. In Vor-Covid-Zeiten galten bundesweit zu speisende Datenbanken als No-Go. Dies bekam vor allem ELGA zu spüren, dessen Ursprungskonzept ein zentrales Datenregister am Plan hatte. Proteste von Datenschützern aus NGOs, aber auch Bedenken der Ärztekammer wischten das Vorhaben vom Tisch.

Diese Haltung hat sich gewandelt. Das Virus hat den Wunsch nach erhöhter Prognosegenauigkeit und verbesserten Simulationsszenarien in den Vordergrund gepresst. Im Mai hat die türkis-grüne Regierung ein Standortkonzept mit dem Namen „Chancenreich Österreich“ vorgestellt. In dem Zusammenhang werden im Wirtschaftsministerium Überlegungen angestellt, die mittelmäßige Performance Österreichs im Bereich „Digital Health“ zu steigern. Denn einer gut ausgebauten Dateninfrastruktur stehe „ein ernüchternd geringer Grad tatsächlicher

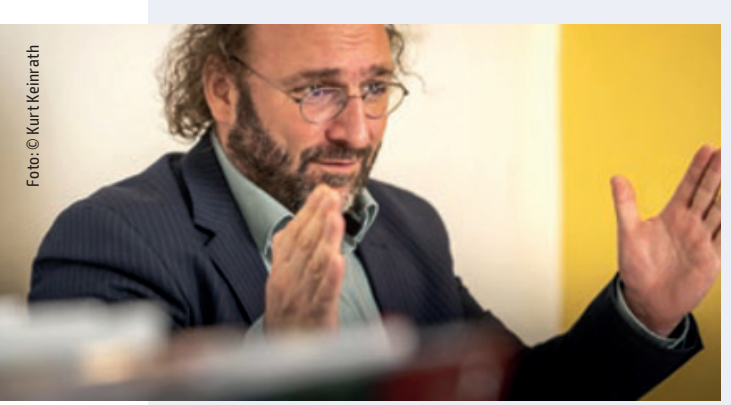

Herr Popper, verhindert der Datenschutz in Österreich eine effektive Pandemieforschung?

Das kann man so nicht sagen. Datenschutz setzt Grenzen, über die ich als Forscher manchmal unglücklich bin, aber als Bürger deren Sinn einsehe. Es sind zwei Dinge, die mir wichtig erscheinen. Erstens müssen die Men- schen, die Daten anfordern, in der Lage sein, diese auch zu verarbeiten. Ich habe den Eindruck, da kommen Leute, die sagen, gebt uns alle eure Daten, aber wir wissen noch nicht so genau, was wir damit tun. Also muss der Forscher über die richtigen Methoden verfügen. Faktum ist: In der Datenforschung wollen die einen immer alles, ohne genau sagen zu können, was sie damit bezwecken. Und die anderen machen sich grundsätzlich Sorgen.

Die Wissenschaft benötigt rechtskonform aufbereitete Daten. Dabei geht es in erster Linie um Anonymisierung. Schaffen die Behörden das?

Die Aufbereitung der Daten zu Forschungszwecken ist sicher ein Flaschenhals. Da ist die Perfomance sehr unterschiedlich. Manchmal scheitert es an den eigenen Strukturen der Institutionen. Manchmal sind es auch die rechtlichen Rahmenbedingungen, die nicht auf den Boden gebracht werden. Leider gibt es keine normierten Vorgaben für Aufbereitung und Anonymisierung von Daten. Und das ist das Problem. Wir haben mit dem K-Projekt
Dexhelpp genau diese Methoden entwickelt. Daten werden auf einer virtuellen Maschine so verschnitten, dass sie $\mathrm{K}$-anonymisiert sind. K-anonymisiert heißt, dass ich unter keinen Umständen Gruppen kleiner drei identifizieren kann. Aber es gibt dazu keine Normen.

In der Statistik Austria sollen künftig im Austrian Micro Data Center (AMDC) die Daten von Behörden zusammengeführt werden, um sie forschenden Institutionen zur Verfügung zu stellen. Soll dieser Datenschatz auch für forschende Unternehmen oder Start-ups zur Verfügung stehen?

Da bin ich skeptisch. Bei der Datenverwendung muss ich mich immer fragen: Cui bono? Ich verstehe, dass Start-ups nicht nur eigene Studien auswerten wollen. Aber man muss auch sagen: Es ist nicht im öffentlichen Interesse, dass die Firma XY mehr Kohle macht, auch wenn dies grundsätzlich nichts Böses ist. Es braucht einen Interessensausgleich: Wenn Unternehmen Daten aus dem öffentlichen Bereich bekommen sollen, dann muss klar sein, dass die Öffentlichkeit profitieren muss. 
Datennutzung gegenüber“, zitiert der „Standard“ aus einem Schreiben aus dem Ressort Margarete Schramböcks. In dem Memo wurde hinterfragt, inwiefern „bestehende Regulatorik und Rechtslagen erweitert und angepasst werden (müssen), um eine umfangreiche Nutzung der Gesundheitsdaten (zum Beispiel ELGA) zu ermöglichen." Derzeit wird hinter den Kulissen diskutiert und ein klein bisschen verhandelt. Vertreter der Sozialpartner, Gesundheitsinstitutionen, aus der Forschung und Wissenschaft, aber auch aus Unternehmen und NGOs deponieren in großem Kreis ihre Vorschläge.

\section{Mutter aller Pandemiedaten}

Statistik - vor allem wenn es um Gesundheitsdaten geht - ist in Österreich in weiten Bereichen Gesetzessache. Es gibt zum Beispiel das Krebsstatistikgesetz, das Krankenanstalten verpflichtet, onkologische Befunde an die Statistik Austria zu melden. Es gibt das Personenstandsgesetz, das die Behörden anweist, Geburt, Ehe, Eingetragene Partnerschaft oder Tod an das Zentrale Personenstandsregister zu melden. Und es gibt das Epidemiegesetz, dessen Paragraph 4 vorschreibt, sämtliche anzeigenpflichtige Infektionserkrankungen bei den zuständigen Bezirksverwaltungsbehörden anzuzeigen.

Das Epidemiologische Meldesystem EMS führte bis Februar des vorigen Jahres ein Dornröschendasein: Ein paar Masern, etwas Röteln und Scharlach, infektiöse Hepatitis, Tuberkulose - mehr gab es in dem Register bis Anfang 2020 selten zu entdecken. Seit einer Novelle im März 2020 ist das EMS die Goldader unter den heimischen Datenminen. Damit führt das Register aktuell die Rangliste der Gesundheitsstatistiken an. Auf den Plätzen folgen die Datensätze der Gesundheit Österreich GmbH (GÖG), des Hauptverbands der Sozialversicherungsträger und was Gesundheitsdaten angeht - mit einigem Abstand auf Platz 4 der etatmäßige Datenchampion, die Statistik Austria.

Die Daten des EMS werden parallel in ein aufbereitetes Statistikregister überführt, das der wissenschaftlichen Forschung dient. Darin werden seit Mitte März auch Hospitalisierungsdaten von Patienten mit einer Haupt- oder Nebendiagnose Covid-19 zur Verfügung gestellt. Die Daten enthalten anonymisierte Informationen, in die klinische Angaben gepackt werden. Zusätzlich erfasst werden Daten zum Umfeld des Erkrankten, soweit sie in Bezug zur anzeigepflichtigen Erkrankung stehen, und Daten zu den getroffenen Vorkehrungsmaßnahmen.

Was dabei den Statistikern immer wieder sauer aufstößt: Diese anonymisierten Informationen dürfen - vorerst - aus Datenschutzgründen n i c h t mit anderen Daten kombiniert oder verknüpft werden. Denn damit steigt das „Risiko der Re-Identifikation“, wie Josef Kytir, Leiter der Direktion Bevölkerung in der Statistik Austria erklärt. Er verweist auf das mathematische Faktum, dass anonymisierte Daten mit jeder Verknüpfung ein Stück von ihrem Identitätsschutz verlieren. Je mehr Daten miteinander in Beziehung gesetzt werden, umso näher kommen die Zahlenmenschen der Persönlichkeit des betreffenden Individuums.

Für Forscher wie Niki Popper sind die Verknüpfungsverbote ein massives Problem - und nur bedingt sinnvoll. Daten, die nach „lege arte“-Methoden anonymisiert und geordnet werden, verlieren auch nach mannigfacher Verknüpfung nur wenig von ihrem Geheimnis. Es liegt an der Kompetenz der Datenbankbetreiber, den Zahlensätzen die Identitäten zu nehmen. Dann erfahrt Pandemieforschung in Österreich eine neue Qualität - bei Wahrung aller Rechte.

\section{DIE STILLE REVOLUTION}

Fokussierter Ultraschall hat das Potenzial, Therapie, medizinische Behandlung und auch das Management von Kliniken voranzubringen. Nach Angaben der Focused Ultrasound Foundation, USA, haben die global führenden Unternehmen ihre Investitionen im Jahr 2020 im Vergleich zum Vorjahr verdreifacht. Die Zahl der Behandlungsstandorte ist weltweit um 36 Prozent auf rund 800 gestiegen. Mittlerweile wurden mehr als 300.000 Patienten mit fokussiertem Ultraschall behandelt, fast die Hälfte davon im Jahr 2019, was die Wachstumsdynamik unterstreicht. Und es kommen laufend neue Anwendungen hinzu. Allein im Bereich neurologischer Indikationen laufen klinische Studien zur Anwendung bei Depressionen, neuropathischen Schmerzen, Bluthochdruck und verschiedenen Krebsarten.

\section{Eine disruptive Alternative und Ergänzung zur Chirurgie}

Fokussierter Ultraschall bietet Patienten und Kliniken Vorteile: Teure OP-Zeiten werden durch weniger kostenintensive Zeiten am MRT ersetzt. Durch die Vermeidung des chirurgischen Schnitts verläuft die Rekonvaleszenz meist schneller. Für Kliniken kann dies weniger Pflegeaufwand und geringere Kosten bedeuten. Zudem können durch die Aufnahme eines MRgFUS-Programms ins Behandlungsportfolio mehr und bislang unterversorgte Patienten behandelt werden. Zusätzliche Wachstumschancen ergeben sich aus der Tatsache, dass die Technologie gerade erst am Anfang steht.

\section{Wachstumspotenzial für Kliniken}

Allein 1 Million Menschen sind in Deutschland von Tremor betroffen, schätzt die Deutsche Gesellschaft für Neurologie'. Gleichzeitig wächst die Zahl weiterer Anwendungsmöglichkeiten für fokussierten Ultraschall. Während die Senkung der Kosten für die Patientenversorgung die wirtschaftliche Basis der Kliniken stärken kann, bieten sich hierdurch Wachstumschancen. Fokussierter Ultraschall kann damit beides leisten: das Leben der Patienten verbessern und gleichzeitig Krankenhäusern und Kliniken helfen, ihre Führungsposition auszubauen.

\section{Kontakt: Peter Medina}

Director Central Europe \& Netherlands INSIGHTEC

peterm@Insightec.com

1 Diener H-J, Weimar C. Extrapyramidalmotorische Störungen - Tremor. In: Leitlinien für Diagnostik und Therapie in der Neurologie. Stuttgart: Thieme Verlag. 